\title{
A espiritualidade no cuidado paliativo em insuficiência cardíaca, à luz da Teoria de Jean Watson."
}

\author{
Spirituality in palliative care in heart failure, in light of the Theory of Jean Watson. \\ Espiritualidad en cuidados paliativos en insuficiencia cardíaca, a la luz de la Teoría de Jean Watson.
}

Domingos da Silva Manuel ${ }^{1}$, Jacqueline da Silva do Nascimento², Paloma Santana Pereira ${ }^{3}$, Renê dos Santos Spezani ${ }^{4}$

Como citar esse artigo. Manuel, DS; do

Nascimeto, JS; Pereira, PS; Spezani, RS.

A espiritualidade no cuidado paliativo em

insuficiência cardíaca, à luz da Teoria de

Jean Watson. Revista Pró-UniverSUS.

2019 Jul./Dez.; 10 (2): 64-69.

\begin{abstract}
Resumo
Objetivos: Descrever as dificuldades apresentadas pelo enfermeiro para implementar a espiritualidade na assistência ao paciente com insuficiência cardíaca em fase terminal em cuidados paliativos e analisar os desafios que se apresentam na atualidade para o enfermeiro, para a implementação da espiritualidade no cuidado de enfermagem ofertado ao paciente com insuficiência cardíaca em fase terminal, à luz do Referencial Clinical Caritas de Jean Watson. Métodos: Trata-se de pesquisa bibliográfica, com natureza descritiva e abordagem qualitativa. O levantamento de dados foi realizado por meio de busca na Biblioteca Virtual em Saúde (BVS), nas bases de dados da Literatura Latino Americana e do Caribe em Ciências e Saúde (LILACS) e Scientific Electronic Library Online (SCIELO), a partir dos descritores: Espiritualidade; Enfermagem; Insuficiência Cardíaca, Jean Watson. Resultados: Identifica-se que o enfermeiro se sente despreparado, para lidar com pacientes acometidos pela Insuficiência Cardíaca em situações de finitude, tendo que aprender a lidar com a impotência da perda diante da incessante busca pela cura. Conclusão: Há necessidade de programar uma assistência de enfermagem que valorizea espiritualidade do paciente que apresenta Insuficiência Cardíaca e o referencial adotado pode contribuir pra que isso seja efetivado. Sugere-se que a formação dos enfermeiros contemple a oferta de disciplinas que estabeleçam interfaces com a espiritualidade e finitude humana, com vistas a oportunizar melhor preparo, para que estes possam compreender esse processo e,simultaneamente, proporcionar uma morte com amparo, acolhimento e maior dignidade aos pacientes em questão.

Palavras-chave: Cuidados de Enfermagem, Cuidados Paliativos, Espiritualidade Insuficiência Cardíaca.
\end{abstract}

\begin{abstract}
Objectives: To describe the difficulties presented by nurses to implement spirituality in the care of patients with terminal heart failure in palliative care and to analyze the current challenges facing nurses for the implementation of spirituality in nursing care offered to patients. With terminal heart failure in the light of Jean Watson's Clinical Caritas Methods: This is a bibliographical research, with descriptive nature and qualitative approach. Data collection was performed by searching the Virtual Health Library (VHL), the Latin American and Caribbean Science and Health Literature (LILACS) and Scientific Electronic Library Online (SCIELO) databases, using the descriptors : Spirituality; Nursing; Heart Failure, Jean Watson. Results: It is identified that nurses feel unprepared to deal with patients affected by heart failure in terminal situations, having to learn to deal with the impotence of loss in the face of the incessant search for cure. Final considerations: There is a need to program nursing care that values the spirituality of patients with heart failure and the adopted reference can contribute to this. It is suggested that the training of nurses contemplates the offer of disciplines that establish interfaces with human spirituality and finitude, in order to provide better preparation, so that they can understand this process and, at the same time, provide a death with protection, acceptance and greater dignity to the patients in question.
\end{abstract}

Keywords: Nursing Care, Palliative care, Spirituality Heart Failure.

Afiliação dos autores: 1. Bacharel em Enfermagem, Centro Universitário Augusto Motta, Rio de Janeiro, RJ, Brasil. Email: dms000325@gmail.com ORCID: https://orcid.org/00000002-9202-0775

2. Bacharel em Enfermagem, Centro Universitário Augusto Motta, Rio de Janeiro, RJ, Brasil. Email: jacqueline.enfa2019@gmail.com ORCID: https://orcid.org/0000-0003-28860275

3. Bacharel em Enfermagem, Centro Universitário Augusto Motta, Rio de Janeiro, RJ , Brasil. Email: palomamiglu@gmail.com ORCID:Orcid: https://orcid.org/0000-0002-50657655

4. Pós-doutorando em Enfermagem - UERJ. Professor do Centro Universitário Augusto Motta, Rio de Janeiro, RJ, Brasil. Email: renespezani@gmail.com ORCID:Orcid: https:// orcid.org/0000-0002-5347-6112 


\section{Resumen}

Objetivos: Describir las dificultades que presentan las enfermeras para implementar la espiritualidad en la atención de pacientes con insuficiencia cardíaca terminal en cuidados paliativos y analizar los desafíos actuales que enfrentan las enfermeras para la implementación de la espiritualidad en la atención de enfermería ofrecida a los pacientes. con insuficiencia cardíaca terminal a la luz de la Clínica Caritas de Jean Watson Métodos: se trata de una investigación bibliográfica, con carácter descriptivo y enfoque cualitativo. La recopilación de datos se realizó buscando en las bases de datos de la Biblioteca Virtual en Salud (BVS), la Literatura de Ciencia y Salud de América Latina y el Caribe (LILACS) y la Biblioteca Electrónica Científica en Línea (SCIELO), utilizando los descriptores: Espiritualidad; Enfermería; Insuficiencia cardíaca, Jean Watson. Resultados: Se identifica que las enfermeras no se sienten preparadas para tratar con pacientes afectados por insuficiencia cardíaca en situaciones terminales, teniendo que aprender a lidiar con la impotencia de la pérdida ante la búsqueda incesante de cura. Consideraciones finales: Existe la necesidad de programar la atención de enfermería que valore la espiritualidad de los pacientes con insuficiencia cardíaca y la referencia adoptada puede contribuir a esto. Se sugiere que la formación de enfermeras contemple la oferta de disciplinas que establezcan interfaces con la espiritualidad y la finitud humanas, a fin de proporcionar una mejor preparación, para que puedan comprender este proceso y, al mismo tiempo, proporcionar una muerte con protección, aceptación y mayor dignidad a los pacientes en cuestión.

Palabras clave: Atención de enfermería, Cuidados paliativos, Espiritualidad Insuficiencia cardíaca.

\section{Introdução}

Esse estudotem por objeto a espiritualidade no contexto dos cuidados paliativosprestados ao paciente com Insuficiência Cardíaca, à luz do referencial Clinical Caritas de Jean Watson.

A motivação para a escolha do objeto emerge da vivência acadêmica dos pesquisadores, onde foi possível perceber que existem lacunas no que se reporta ao ensino dos cuidados paliativos e da espiritualidade na formação de graduandos em enfermagem que precisam ser alvo de reflexões e debates, com vistas a viabilizar uma assistência realmente holística e humanizada aos pacientes que vivenciam doenças crônicas e terminais.

A insuficiência cardíaca pode ser definida como uma síndrome clínica resultante de um distúrbio cardíaco funcional ou estrutural que prejudica a capacidade do ventrículo suprir o aporte sanguíneo adequado de acordo com a demanda do corpo, dificultando as atividades e incapacitando funcionalmente o individuo acometido por insuficiência cardíaca ${ }^{1}$.

Os cuidados paliativos são extremamente importantes para a assistência ao portador de insuficiência cardíaca, devendo abranger o controle de sinais com sem negligenciar a garantia de suporte emocional e espiritual ${ }^{2}$.

Os cuidados paliativos devem ser realizados por uma equipe multidisciplinar, tendo por finalidade a melhoria da qualidade de vida do paciente e também a de seus familiares, diante de uma doença que ameace a vida, por meio da prevenção e alívio do sofrimento, da identificação precoce, avaliação e tratamento de dor e demais sintomas físicos, sociais, psicológicos e espirituais ${ }^{3}$.

Na literatura, há endosso de que a valorização da espiritualidade é de extrema importância na esfera dos cuidados paliativos, pois é da natureza do ser humano buscar por conforto e por uma força maior que lhe dê esperança ou apenas o auxilie a aceitar e enfrentar melhor as situações a iminência de morte ou mesmo a morte em si ${ }^{4}$.
Relata-se que a espiritualidade se refere"à busca pessoal para compreensão das questões finais sobre a vida e sua relação com o sagrado e o transcendente, que pode ou não levar ao desenvolvimento de práticas religiosas" . Dessa forma, o paciente pode buscar a espiritualidade como forma de enfrentamento de doenças, com a finalidade de minimizar o sofrimento decorrente das dificuldades encontradas ou para obter maior esperança de cura com o tratamento ${ }^{6}$.

Essa tendência vai ao encontro das concepções de Jean Watson, uma vez que sua teoria se propõe a ir além dos modelos de cuidado tradicionais da enfermagem. No modelo de cuidado proposto por Watson, o enfermeiro precisa estar aberto para as questões que não se limitem a aspectos objetivos, sendo importante considerar as concepções, as crenças, os valores e a espiritualidade de quem é cuidado 7 .

Margaret Jean Watson desenvolveu a Teoria do Cuidado Humano, que considera o cuidado efetivo por meio do relacionamento transpessoal, ou seja, o cuidado que transcende tempo, espaço e matéria de paciente e profissional para que formem um único elemento em sintonia, além do momento pontual da interação, de maneira a favorecer a restauração ${ }^{8}$.

$\mathrm{O}$ paciente com insuficiência cárdica vivencia conflitosinternos quemuitasvezesserefletemdiretamente em sua espiritualidade. Esses clientes necessitam de uma assistência de enfermagem humanizada por meio da qual se respeite sua individualidade, para que lhes seja proporcionado um fim de vida com dignidade ${ }^{2}$. Logo, o respeito à crença dos pacientes e a estimulação para que este mantenha viva sua espiritualidade são importantes para que possam ser cuidados com conforto e para que aceitem melhor o processo que está sendo vivenciado $^{2,8}$.

De acordo com os estudos analisados ${ }^{5,6,7}$, percebese que este cuidado humanizado frente à finitude já vem sendo aplicado em muitas instituições de saúde e com resultado satisfatório, perante a equipe de enfermagem e principalmente para o paciente, que é o foco principal.

Espera-se que esse estudo possa contribuir 
para a pesquisa, ensino e assistência em enfermagem, respectivamente, ao ampliar a produção científica existente sobre a temática e suscitar, simultaneamente, argumentos necessários à consumação de novas formas de pensar e fazer, com vistas oferta de um cuidado paliativo efetivamente integral e humanizado aos pacientes que vivem com insuficiência cardíaca.

Dessa forma, considerando o que foi exposto, os objetivos do estudo consistem em: descrever as dificuldades apresentadas pelo enfermeiro para implementar a espiritualidade na assistência ao paciente com insuficiência cardíaca em fase terminal em cuidados paliativos; analisar os desafios que se apresentam na atualidade para o enfermeiro para a implementação da espiritualidade no cuidado de enfermagem ofertado ao paciente com insuficiência cardíaca em fase terminal à luz do Referencial Clinical Caritas de Jean Watson.

\section{Metodologia}

Trata-se de pesquisa bibliográfica, com natureza descritiva e abordagem qualitativa.

A busca de dados foi realizada em acervo virtual, na Biblioteca Virtual em Saúde (BVS). Para tanto, recorreu-se aos bancos de dados da Literatura Latino Americana e do Caribe em Ciências e Saúde (LILACS) e Scientific Electronic Library Online (SCIELO), sendo utilizados ostermos indutores espiritualidade; cuidados de enfermagem; insuficiência Cardíaca, Teoria do Cuidado Transpessoal de Jean Watson.

Para a seleção dos dados, foram estabelecidos critérios de inclusão e exclusão: Os critérios de inclusão estabelecidos foram: produções científicas disponibilizadas em periódicos online com textossocializados na íntegra para leitura; publicadas no recorte temporal compreendido no período de 2010 a 2019, na língua portuguesa e que abordassem a problemática do presente estudo. Foram excluídos da busca os materiais duplicados e que não corroboravam com o alcance dos objetivos da pesquisa.

Diante dos critérios adotados, foram selecionadas 18 produções, as quais foram analisadas em conformidade com o que preconiza Bardin ${ }^{9}$ e seus resultados posteriormente organizados e apresentados em categorias temáticas.

\section{Resultados e discussão}

Em conformidade com o desenho metodológico adotado, revelaram-se relevantes para o alcance dos objetivos as seguintes categorias:dificuldades apresentadas pelo enfermeiro para implementar a espiritualidade na assistência ao paciente com insuficiência cardíaca em fase terminal em cuidados paliativos e desafios que se apresentam na atualidade para o enfermeiro para a implementação da espiritualidade no cuidado de enfermagem ofertado ao paciente com IC em fase terminal à luz do Referencial Clinical Caritas de Jean Watson.

\section{Dificuldadesapresentadaspeloenfermeiro para implementar a espiritualidade na assistência ao paciente com insuficiência cardíaca em fase terminal em cuidados paliativos}

Verifica-se que a finitude humana requisita um cuidado mais complexo, uma vez que o paciente que vivencia essa fase da vida carece de uma assistência que seja capaz de contemplar todas as suas necessidades biopsicossocioespirituais ${ }^{10}$.

Nesse contexto, relata-se que diante das situações que coadunam com a finitude humana, o exercício da espiritualidade é essencial para o seguimento da vida das pessoas em que se encontram sob assistência paliativa, podendo ser considerada uma força motriz para dar respostas aos mais verdadeiros desejos desses sujeitos em sua relação exixtencial ${ }^{11,12}$.

Contudo, apesar de sua consagrada importância, observa-se a existênciade dificuldades que limitam a implementação da espiritualidade nas práticas assistenciais de enfermagem ${ }^{5,13}$. Essas dificuldades emergem a partir da formação acadêmica de enfermeiros, onde conteúdos que versam sobre o tema nem sempre são abordados nas unidades curriculares dos cursos de graduação. Desse modo, é importante que essas deficiências sejam alvo de empreendimentos que modifiquem esse panorama, considerando a relevância do enfoque holístico que deve ser ofertado ao paciente nesse contexto e a própria contribuição da espiritualidade sobre a finitude de vida ${ }^{13}$.

Através da literatura, verifica-se que a enfermagem se sente despreparada para prestar assistência ao paciente em fase terminal, fugindo muitas vezes de discussões sobre o tema, com receio de ser mal interpretada ou de sofrer com o debate ${ }^{14}$.

Por outro lado, situações como estas ocorrem pois os enfermeiros aprendem desde cedo a preservar a vida e a buscar a cura, sem serem estimulados a refletir sobre a morte ${ }^{13}$. Assim, os sentimentos de impotência e de fracasso gerados em consequência desse contexto, induzem, muitas vezes, um modo de agir protetivo contra o sofrimento psicológico, que resulta no distanciamento dos pacientes, que tanto necessitam desse cuidado ${ }^{14}$.

Além das deficiências advindas da formação, existem outros fatores que dificultam a implementação de uma assistência que contemple a espiritualidade na esfera paliativa, como a falta de tempo, privacidade e confiança ${ }^{5}$. Dessa forma, percebe-se que além das 
deficiências apontadas na formação, muitos enfermeiros vivenciam dificuldades diárias quando se deparam com pacientes que enfrentam doenças sem possibilidade de cura, em boa parte das situações porque isso é acirrado em decorrência das atribuições que lhes são impostas do dia a dia e que lhes distanciam da possibilidade de identificar suas reais necessidades.

Sabe-se que os profissionais muitas vezes trabalham em meio uma grande sobrecarga, com escalas apertadas e um número grande de pacientes. Dessa forma, essas condições culminam por dificultar a projeção de um olhar humanizado e holístico, que o paciente neste estágio da vida necessita. Aliada a esses fatores, também se constata a dificuldade de contemplara família nesse processo, a qual pode encarar a ausência da abordagem da espiritualidade como negligência da equipe $^{11}$.

Essas constatações propiciam espaços para a reflexão sobre o fato de que a morte, apesar de natural à existência humana, ainda configura, em pleno século XXI, uma difícil missão a ser aceita pela humanidade, incluindo os próprios enfermeiros. Igualmente, possibilitam a aposição de reflexões acerca do processo de formação, visto que reportam à inferência de que muitos dentre os profissionais de enfermagem aprendem em sua formação, que devem buscar de todas as maneiras a cura, muitas vezes pautados em uma visão biomédica e determinista da saúde, deixando os espaços de formação com a falsa compreensão de que os cuidados paliativos configuram um fracasso da equipe de saúde, quando na verdade não o são.

Embora os profissionais de enfermagem identifiquem a existência dessas carências, verifica-se que muitos buscam superar essas lacunas, na tentativa de realizar um cuidado humanizado, que contribua para oportunizar uma melhor qualidade de vida durante o processo que envolve a finitude ${ }^{14}$.

Desse modo, é fundamental que o enfermeiro esteja preparado para planejar uma assistência que contemple a espiritualidade, especialmente no contexto da insuficiência cardíaca. Esse preparo, portanto, deve abranger questões vinculadas ao cuidado integral ao paciente e sua família, com vistas a promover conforto físico, espiritual e criação de um ambiente de aceitação e enfrentamento.

Considerando que boa parte dos currículos de formação de graduandos em enfermagemenfatizamprocedimentos técnicos e o cuidado com o corpo físico do paciente, deixando lacunas no que se reporta às necessidadesde atendimento psicológico aos pacientes terminais e suas famílias, muitos dentre esses graduandos apresentam dificuldades para lidar com a morte e com as questões a ela vinculadas ${ }^{10}$.

Diante destes apontamentos, observa-se a necessidade de dedicar mais estudos, e inserir desde a graduação, temáticas que envolvam a abordagem da espiritualidade como ferramenta de cuidado a pacientes que vivenciam afinitude, visto que, dessa forma, possivelmente serão ampliadas as chances de formação de profissionais com maior qualificação para lidar com esses pacientes ${ }^{5,13}$.

Como a insuficiência cardíaca atinge um quantitativo significativo de pacientes em todo o mundo, não é difícil que um enfermeiro se depare com um paciente em cuidado paliativo enfrentando os desdobramentos dessa doença. Logo, esse profissional deve estar atento e preparado para implantar, junto ao paciente e seus cuidadores, ações de paliação que os contemplem de maneira holística e humanizada. Nesta ocasião, o alívio dos sintomas, a melhora da qualidade de vida, o apoio emocional, a detecção e o tratamento precoce das descompensaçõesdevem ser priorizados e constituem desafios à prática profissional de enfermagem contemporânea, a qual deve conferir segurança, qualidade e resultado ao que se espera acerca de sua atuação junto aos usuários em questão ${ }^{15}$.

\section{Desafios que se apresentam na atualidade para o enfermeiro para a implementação da espiritualidade no cuidado de enfermagem ofertado ao paciente com insuficiência cardíaca em fase terminal à luz do referencial Clinical Caritas de Jean Watson}

As teorias de enfermagem contribuem para fundamentar a prática e sistematizar a assistência de enfermagem ${ }^{15}$. Nessa perspectiva, uma das teorias mais utilizadas na atualidade para nortear o agir da enfermagem com vistas a oportunizar um atendimento mais atento e humanizado aos pacientes e familiares é a Teoria do Cuidado Humano de Jean Watson ${ }^{16}$.

De acordo com esse referencial teórico-filosófico, entende-se que a para que seja estabelecida uma boa relação de cuidado, enfermeiros e pacientes necessitam uns dos outros, fortalecem sua união e setornam capazes de estabelecer a transpessoalidade,elevando a consideraçãopelo seu modo de estar no mundo8.

O Clinical Caritas é a denominação do modelo teórico de cuidado atual e aprimorado, inicialmente proposto por Watson, em que se abarcam os seguintes elementos: prática da bondade e equanimidade;presença e valorizaçãodo sistema de crenças do ser cuidado; cultivo de práticas espirituais próprias, aprofundando o conhecimento individual; manutenção de um cuidado autêntico por meio de um relacionamento de ajuda-confiança; apoio à expressão de sentimentos positivos e negativos; utilização de conhecimento e intuição de forma criativa na resolução de problemas; 
estabelecimento de vínculos verdadeiros na experiência de ensino- -aprendizagem; oferta de um ambiente de restauração física, emocional e espiritual; promoção de alinhamento de corpo, mente e espírito, com vistas atender às necessidades do indivíduo; consideração de aspectos espirituais e de vida e morte ${ }^{16}$.

Como o cuidado ao paciente em finitude é bem peculiar, cabe aos enfermeiros, que são responsáveis pelo planejamento da assistência, conhecer as características da espiritualidade do indivíduo e também como ele pode necessitar de sua utilização durante o enfrentamento da doença ${ }^{13}$.

Nesse sentido, aprender a lidar com as perdas em um ambiente onde a busca pela cura predomina é desafiador, e poucos estão dispostos a discutir e enfrentar esse aspecto inerente ao cuidado. Para a enfermagem oferecer os cuidados paliativos e abordar questões espirituais, é necessário que os profissionais compreendam essa vivência como um compartilhamento terapêutico de momentos de amor e compaixão, sustentando a ideia de que é possível tornar a morte digna e assegurando que o paciente receba o suporte e o acolhimento necessário durante esse processo ${ }^{17}$.

Entretanto, percebe-se a que estruturação da rede de cuidados paliativos ainda carece de ações eficazes para o seu desenvolvimento, capazes de assegurar uma política pública coerente com os princípios doutrinários do Sistema Único de Saúde. A partir dessa perspectiva, instaura-se, enquanto desafio a ser superado, a necessidade de formação de profissionais de habilitados para a promoção de cuidados paliativos que atendam àsdemandas assistenciais, não só dos pacientes, mas também as de seus respectivos familiares ${ }^{18}$.

Entre os desafios que se apresentam, há a necessidade das instituições de ensino, assim como os hospitais, investirem na capacitação de seus profissionais para ofertar um cuidado integral, respeitando e ao mesmo tempo, promovendo junto ao paciente e família, a valorização de sua espiritualidade, o conforto, a dignidade e a serenidade para que se tornem mais fortalecidos e preparados para vivenciar o processo de finitude.

Nesse caso, desafia-se o enfermeiro para que também trabalhe com sua equipe, no sentido de contemplar a espiritualidade ao assistir pessoas que vivenciam o processo de finitude de maneira empática, considerando, inclusive, a necessidade de estabelecimento de oportunidades para que possam dialogar e compartilhar suas experiências e dificuldades relacionadas a esse modelo de cuidado.

A programação de uma assistência voltada para o cuidado espiritual e humanizado diante da finitude é essencial, uma vez que pode contribuir para uma melhoria da qualidade da assistência prestada ${ }^{19}$. Logo, a oferta de disciplinas nos cursos de formação profissional e o aumento do número de estudos que versem sobre questões relacionadas èespiritualidade e finitude humana possivelmente podem contribuir para que ocorra uma maior compreensão por parte da equipe de enfermagem sobre essas questões.

A aplicação de componentes teóricos e metodológicos nos cuidados de enfermagem é fundamental para que a Enfermagem se fortaleça como profissão e ciência. Dessa forma, também é desafiadora aarticulaçãodo conhecimento produzido baseado em evidências científicas com instrumentos teóricose novas tecnologias disponíveis para o cuidado ${ }^{20}$.

Para cuidar de forma transpessoal, o profissional é desafiado aimplementar ações decididamente transformadoras, por meio das quais todos os envolvidos nas relações que se estabelecem entre quem cuida e quem é cuidado sejam movidos em direção a um elevado sentido de harmonia entre suas mentes, corpo e espírito. $\mathrm{O}$ cuidado transpessoalliberta sentimentos, permitindo que cuidadores e usuários, assimilem melhor suas capacidades, reais condiçõese possibilidades diante de suas formas de ser e estar no mundo ${ }^{8,16,20}$.

Existem várias instituições de saúde na atualidade que utilizam a Teoria do Cuidado Humano, com estudos demonstrando sua real eficácia e a importância. O referencial Clinical Caritas de Jean Watson pode nortear os elementos que devem ser abordados durante o cuidado frente à finitude. Ele ajuda o profissional a abordar o cuidado integral e identificar quaissão, de fato, as reais necessidades que o paciente apresenta num dado momento ${ }^{19}$

Nesse sentido, há um desafio premente que caracteriza uma necessidade de se investir em novas estratégias de atuação, para que esse referencial possa ser efetivamente implementado para aumentar ainda mais a qualidade da atenção no local de trabalho e garantir que todos os profissionais de saúde tenham habilidades necessárias para demonstrar de forma produtiva o cuidado transpessoal ${ }^{20}$.

\section{Conclusão}

$\mathrm{O}$ estudo remete à certeza de que o enfermeiro encontra dificuldades para lidar com a espiritualidade do paciente com insuficiência cardíaca. Essas dificuldades envolvem aspectos relacionados ao processo de aceitação por parte do paciente e seus familiares, a capacitação insuficiente para abordar o assunto e também ao número pequeno de pesquisas que abordam a temática.

Nos estudos que serviram como base para o desenvolvimento dessa pesquisa, destacam-se conflitos acerca da espiritualidade nos momentos de finitude, pouca abordagem da aceitação do paciente e dos familiares e a necessidade de capacitação dos profissionais para melhor abordagem do assunto. Percebe-se que é de suma importância que o debate 
sobre a espiritualidade, cuidados paliativos e modelos teóricos que os contemplem façam parte das unidades curriculares dos cursos de graduação em enfermagem, para que os enfermeiros já se insiram no mercado de trabalho capacitados para lidar com essas respectivas questões.

A insuficiência cardíaca é uma doença que causa um grande impacto na vida do paciente e deseus familiares, propiciando muitas restrições e debilitando o mesmo. Portanto, é fundamental que o enfermeiro atue de maneira empática, com vistas a abrandar os sintomas que dela decorrem e planejar uma assistência que contribua para que ocorra melhora na qualidade de vida. Nesse sentido, é importante lembrar que ações de conscientização da população com relação ao cuidado à saúde cardíaca poderão melhorar a qualidade de vida dos mesmos e reduzir fatores de risco para insuficiência cardíaca.

Apesar de enfermeiro ter consciência de que todos os pacientes deverão passar pelo processo de finitude e que a espiritualidade é de extrema importância para o enfrentamento desse momento, muitos ainda têm dificuldade para lidar com estes pacientes e que vários desafios precisam ser superados para a otimização de uma assistência integral.

Diante destes apontamentos, conclui-se que o referencial adotado pode contribuir para que isso seja efetivado, ratificando-se seu valor heurístico diante do que foi abordado. Para tanto, sugere-se que o processo de formação dos enfermeiros contemple a oferta de disciplinas que estabeleçam interfaces com a espiritualidade e finitude humana, com vistas a oportunizar um maior preparo, para que estes possam compreender esse processo e, simultaneamente, proporcionar uma morte com amparo, acolhimento e maior dignidade aos pacientes em questão.

\section{Referências}

1. Pereira DAG. et al. Capacidade funcional de indivíduos com insuficiência cardíaca avaliada peloteste de esforço cardiopulmonar e classificação da New York Heart Association.Fisioterapia e Pesquisa. 2012; 19 (1): $52-56$

2. Trotte LAC, Caldas CP, Guimarães TCF. Insuficiência cardíaca e cuidados paliativos, uma nova dimensão de cuidado para enfermagem brasileira. Anais do $16^{\circ}$ SENPE; 19 a 22 de junho de 2011; Campo Grande, Mato Grosso. Brasil. [internet]. 2011. [citado em 12 de junho de 2019]. Disponível em: http://www.abeneventos.com.br/16senpe/senpe-trabalhos/ files/0267.pdf

3. Carvalho RT, Parsons HA. Manual de cuidados paliativos ANCP ampliado e atualizado. $2^{\text {a }}$. Ed. São Paulo: Academia Nacional de Cuidados Paliativos; 2012.

4. Silva DIS. Significados e práticas da espiritualidade nocontexto dos cuidados paliativos em pacientes oncológicos adultos. Revista HCPA. 2011; 31(3): 353-8.

5. Evangelista CB, Lopes MEL, Costa SFG, Batista PSS, Batita JBV, Oliveira AMM. Cuidados paliativos e espiritualidade: revisão integrativa da literatura. Revista Brasileira de Enfermagem. 2016; 69(3):591-601.
6. Guerrero GP, Zago MMF, Sawada NO, Pinto MH. Relação entre espiritualidade e câncer: perspectiva do paciente. Revista Brasileira de Enfermagem. 2011; 19 (1): p. 53-9.

7. Gomes IM, Silva DI, Lacerda MR, Mazza VA, Méier MJ, Mercês NNA. Teoria do cuidado transpessoal de Jean Watson no cuidado domiciliar de enfermagem à criança: uma reflexão. Revista Escola de Enfermagem Anna Nery. 2013; 17 (3): 555-561.

8. Favero L, Pagliuca LMF, Lacerda MR. Cuidado transpessoal em enfermagem: uma análise pautada em modelo conceitual. Revista Escola de Enfermagem USP. 2013; 47 (2): 500-5

9. Bardin L. Análise de conteúdo. Lisboa: Edições 70; 2009. 232p.

10. Vieira AT, Oliveira M, Martins ERC, Costa CMA, Alves RN, Marta CB. Cuidado paliativo ao cliente oncológico: percepções do acadêmico de enfermagem. Revista de Pesquisa Cuidado é FundamentalOnline.[internet]. 2017 [citado em 12 de junho de 2019]; 9 (1): 175-80. Disponível em: http:// www.seer.unirio.br/index.php/cuidadofundamental/article/view/5329/pdf_1

11. Arrieira ICO, Thofehrn MB, Porto AR, Moura PMM, Martins CL, Jacondino MB. Espiritualidade nos cuidados paliativos: experiência vivida de uma equipe interdisciplinar. Revista Escola EnfermagemUSP.[internet]. 2018 [citado em 12 de agosto de 2019]; 52:e03312: 1-8. Disponível em: http://www.scielo.br/pdf/reeusp/v52/0080-6234-reeusp-S1980220X2017007403312.pdf

12. Gomes, MP, Souza FBA, Gomes AMT, Silva FGA, Barbosa DJ, Silva ALB. Ressignificação da existência e do cotidiano. Revista Pró-UniverSUS. [internet]. 2019 [citado em 12 de agosto de 2019]; 10 (1): 2-6. Disponível em: http://editora.universidadedevassouras.edu.br/index.php/RPU/article/ view/1712

13. Carvalho GDA, Acioly CMC, Santos SR, Lima OBA, Alves AMPM, Valdevino SC. Dificuldades de enfermeiros assistenciais frente à espiritualidade em cuidados paliativos. Revista Enfermagem UFPE OnLine. [internet]. 2013 [citado em 12 de junho de 2019]; 7 (12): 6814-20. Disponível em: https://periodicos.ufpe.br/revistas/revistaenfermagem/article/.../15061

14. Hermes HR, Lamarca ICA. Cuidados Paliativos: uma abordagem a partir das categorias profissionais de saúde. Ciência \& SaúdeColetiva.2013; 18 (9): 2577-88., 2013. Disponível em <http:/dx.doi.org/10.1590/S141381232013000900012 .

15. Comitê Coordenador da Diretriz de Insuficiência Cardíaca. Diretriz Brasileira de Insuficiência Cardíaca Crônica e Aguda. Arquivos Brasileiros de Cardiologia. 2018; 111 (3): 436-539.

16. Savieto RM, Leão ER. Assistência em enfermagem e Jean Watson: uma reflexão sobre a empatia. Revista. Escola Enfermagem Anna Nery. 2016; 20(1):198-202.

17. Monteiro FF, Oliveira M, Vall J. A importância dos cuidados paliativos na enfermagem. Rev Dor. 2010; 11 (3):.242-248.

18 - Mendes EC, Vasconcellos LCF. Cuidados paliativos no câncer e os princípios doutrinários do SUS. Saúde Debate.2015; 39 (106): 881-892.

19. Watson J. Watson J. Caritas Nursing: Taking time/Making time [gravação de video]. John C Lincoln North Mountain Hospital, Phoenix, EUA; $2012.70 \mathrm{~min}$

20. Tonin L. Aplicação do modelo de cuidado transpessoal em enfermagem domiciliar às crianças com necessidades especiais de saúde e suas famílias [dissertação]. Curitiba: Universidade Federal do Paraná; 2018. 